\title{
NOTES ON COVERING TRANSFORMATION GROUPS
}

\section{S. KINOSHITA ${ }^{1}$}

1. Introduction. Let $K$ and $\widetilde{K}$ be topological spaces. A continuous $\operatorname{map} p$ of $\tilde{K}$ onto $K$ is called a covering map if for any point $a$ of $K$ there exists an open neighborhood $W$ of $a$ such that $p^{-1}(W)$ is a union of disjoint open sets $W_{\alpha}$ and each $p \mid W_{\alpha}$ is a homeomorphism of $W_{\alpha}$ onto $W . \widetilde{K}$ is called the covering space of $K$ of $p$. A covering transformation is an autohomeomorphism $g$ of $\tilde{K}$ such that $p g(x)=p(x)$ for every $x$ of $\tilde{K}$. The covering transformation group of $p$ is the set of all covering transformations with multiplication, which is the composite of transformations. A transformation group $G$ of a topological space $X$ is said to be properly discontinuous if for any $x \in X$ there is a neighborhood $U$ of $x$ such that for any $g \in G, g(U) \cap U=\varnothing$, where $g \neq e$. (Hence for any distinct $g_{1}$ and $g_{2}$ of $G, g_{1}(U) \cap g_{2}(U)=\varnothing$.) Let $X$ be a connected topological space. Then a transformation group $G$ of $X$ is a covering transformation group of the projection map $p: X \rightarrow X / G$ if and only if $G$ is properly discontinuous, where $X / G$ is a topological space (see $[1])$. In this meaning, proper discontinuity characterizes the covering transformation group, but generally $X / G$ may not be a Hausdorff space, while $X$ is a manifold (see $\S 4$ ).

The purpose of this note is to introduce a condition, the Sperner's condition, ${ }^{2}$ about $G$ such that whenever $X$ is a manifold, $X / G$ is also a manifold. We may say that a transformation group $G$ of a topological space $X$ is said to satisfy the Sperner's condition if for any compact subset $C$ of $X$ the set

$$
G[C]=\{g \in G \mid g(C) \cap C \neq \varnothing\}
$$

is finite. A transformation group $G$ of a topological space $X$ is called fixed point free if any $g$ of $G(g \neq e)$ has no fixed point. Then the main theorem of the note is as follows.

THEOREM. The covering transformation group $G$ of a covering map $p$ of a connected topological space $\tilde{K}$ onto a locally compact Hausdorff space $K$ is fixed point free and satisfies the Sperner's condition. Conversely, if $G$ is a fixed point free transformation group of a connected locally com-

Received by the editors January 30, 1967.

${ }^{1}$ Supported partly by NSF grant GP-5458.

2 The condition introduced in the paper is slightly different from the original condition defined by Sperner [2]. A much more similar condition is defined in [3]. See also [4]. 
pact Hausdorff space $X$ satisfying the Sperner's condition, then the projection map $p: X \rightarrow X / G$ is a covering map and $X / G$ is a locally compact Hausdorff space. Further $G$ is the covering transformation group of $p$.

Also a similar statement is true for a connected manifold, where a manifold is a separable, locally Euclidean Hausdorff space.

2. Let $p$ be a covering map of a topological space $\widetilde{K}$ onto a topological space $K$. It is clear that if $K$ is a Hausdorff space, then so is $\tilde{K}$. If $K$ is locally compact, so is $\widetilde{K}$ and if $K$ is regular, so is $\widetilde{K}$.

If $\tilde{K}$ is connected, then any covering transformation group $G$ is properly discontinuous. Therefore $G$ is fixed point free.

To complete the proof of one direction of the main theorem, let us begin with a lemma. Let $K$ be a Hausdorff space and $p$ a covering map of $\tilde{K}$ onto $K$. Let $a$ be a point of $K$ and $W$ a neighborhood of $a$ defined in $\S 1$. Assume that $U$ is a neighborhood of $a$ such that $\bar{U} \subset W$. For each $W_{\alpha}$ choose a point $b_{\alpha} \in W_{\alpha} \cap p^{-1}(U)$. Put $B=\left\{b_{\alpha}\right\}$.

Lemma. $B$ has no limit point, i.e., there is no point $b$ of $\widetilde{K}$ such that for any neighborhood $V$ of $b,(V-b) \cap B \neq \varnothing$.

Proof. Suppose on the contrary that $B$ has a limit point $b$. First we prove that $p(b) \in \bar{U}$. Let $V$ be a neighborhood of $p(b)$. Since $p$ is continuous, there is a neighborhood $V_{1}$ of $b$ such that $p\left(V_{1}\right) \subset V$. Let $b_{\alpha} \in\left(V_{1}-b\right) \cap B$. Then $p\left(b_{\alpha}\right) \in U$, and therefore $V \cap U \neq \varnothing$. Hence $p(b) \in \bar{U}$.

Now $b$ is contained in some $W_{\alpha}$. But since only one $b_{\alpha}$ is contained in $W_{\alpha}, b$ is not a limit point of $B$.

Lemma. The covering transformation group $G$ of $p$, where $p$ is a covering map of a connected topological space $\widetilde{K}$ onto a regular space $K$, satisfies the Sperner's condition.

Proof. Suppose on the contrary that there is a compact subset $C$ of $\tilde{K}$ such that $G[C]$ is not finite. For each $g_{\beta} \in G[C]$ choose a point $b_{\beta} \in g_{\beta}(C) \cap C$. Put $a_{\beta}=g_{\beta}^{-1}\left(b_{\beta}\right)$. Then $a_{\beta} \in C$. Hence there is a point $a$ of $C$ such that for any neighborhood $V$ of $a$ the set $\left\{g_{\beta} \in G[C] \mid a_{\beta} \in V\right\}$ is not finite. Let $W$ be a neighborhood of $p(a)$ defined as in $\$ 1$ and $U$ a neighborhood of $p(a)$ such that $\bar{U} \subset W$. Put $U_{\alpha}=p^{-1}(U) \cap W_{\alpha}$ for each $a$. Then $U_{\alpha} \cap U_{\alpha^{\prime}}=\varnothing$ for any $a \neq a^{\prime}$. Let $a \in W_{\gamma}$. For each $g_{\beta} \in G[C]$ with $a_{\beta} \in U_{\gamma}, b_{\beta}$ is contained in a $U_{\beta}$. Obviously the correspondence of such $g_{\beta}$ to $U_{\beta}$ is one to one. Hence the set $\left\{b_{\beta} \mid g_{\beta} \in[C], a_{\beta} \in U_{\gamma}\right\}$ has no limit point by the above lemma. On the other hand, we have assumed that $b_{\beta} \in C$ and $\left\{g_{\beta} \mid b_{\beta} \in G[C]\right.$, $\left.a_{\beta} \in U_{\gamma}\right\}$ is not finite, which is a contradication. 
We may note that any locally compact Hausdorff space is regular. Therefore, the proof of one direction of the main theorem is completed.

3. LemMA. Let $G$ be a fixed point free transformation group of a locally compact Hausdorff space $X$. If $G$ satisfies the Sperner's condition, then $G$ is properly discontinuous.

Proof. Let $U_{1}$ be a neighborhood of $x \in X$ such that $\bar{U}_{1}$ is compact. Since $G\left[\bar{U}_{1}\right]$ is finite, there are only a finite number of $g(x), g \in G$, such that $g(x) \in U_{1}$. Since $G$ is fixed point free, there is a neighborhood $U$ of $x$ such that $\bar{U}$ does not contain any $g(x)(g \neq e)$ and $U \subset U_{1}$.

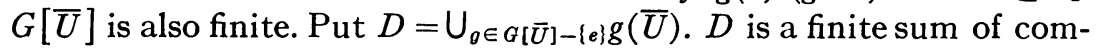
pact sets and hence compact. $D$ does not contain the point $x$. Then there is a neighborhood $V$ of $x$ such that $V \subset U$ and $V \cap D=\varnothing$. Now it is easy to see that $g(V) \cap V=\varnothing$ for any $g \neq e$.

Let $G$ be a fixed point free transformation group of a locally compact Hausdorff space $X$, satisfying the Sperner's condition. Then by the above arguments, the projection $p$ of $X$ onto the orbit space $X / G$ is a covering map. Further if $X$ is connected, then $G$ is the covering transformation group of $p$.

LEMма. $X / G$ is locally compact and Hausdorff.

Proof. It is clear that $X / G$ is locally compact. We have to prove that if $x \neq g(y)$ for any $g \in G$, then there are neighborhoods $U$ and $V$ of $x$ and $y$, respectively, such that $U \cap g(V)=\varnothing$ for any $g \in G$; that is to say, $g_{1}(U) \cap g_{2}(V)=\varnothing$ for any $g_{1}, g_{2} \in G$.

Since $x \neq y$, there are neighborhoods $U_{1}$ and $V_{1}$ of $x$ and $y$ such that $U_{1} \cap V_{1}=\varnothing$ and that both $\bar{U}_{1}$ and $\bar{V}_{1}$ are compact. Put $C=\bar{V}_{1} \cup\{x\}$. Since $G$ satisfies the Sperner's condition, there are only a finite number of elements $g$ of $G$ such that $g(x) \in \bar{V}_{1}$. Then there exists a neighborhood $V$ of $y$ such that $\bar{V} \cap g(x)=\varnothing$ for any $g \in G$ and $V \subset V_{1}$. Now apply the Sperner's condition for $C^{\prime}=\bar{U}_{1} \cup \bar{V}$. Then only a finite number of images of $\bar{V}$ intersects with $\bar{U}_{1}$, and these images $g(\bar{V})$ do not contain the point $x$. Therefore there exists a neighborhood $U$ of $x$ such that $\bar{U} \cap g(\bar{V})=\varnothing$ for any $g \in G$. Hence the proof is completed.

Now, by the above two lemmas, we complete the proof of the other direction of the main theorem.

4. REMARK. Let $h$ be an orientation preserving fixed point free autohomeomorphism of a plane $R^{2}$. Then $h$ induces a topological transformation group $G$, which is infinite cyclic. Such a $G$ is always a properly discontinuous transformation group (see for instance [2]) and hence the projection $R^{2} \rightarrow R^{2} / G$ is a covering map. But $R^{2} / G$ is a 
Hausdorff space if and only if $G$ satisfies the Sperner's condition, hence if and only if $h$ is topologically equivalent to a translation (see $[2])^{3}$. This may justify consideration of a transformation group, which is not only properly discontinuous, but also to satisfy the Sperner's condition.

\section{REFERENCES}

1. E. H. Spanier, Algebraic topology, McGraw-Hill, New York, 1966.

2. E. Sperner, Ueber die fixpunktfreien Abbildungen der Ebene, Abh. Math. Sem. Univ. Hamburg 10 (1934), 1-47.

3. S. Kinoshita, On quasi-translations in 3-space, Fund. Math. 56 (1964), 69-79.

4. D. Montgomery and L. Zippin, Translation groups in three-space, Amer. J. Math. 49 (1937), 121-128.

5. P. S. Mostert, One-parameter transformation groups in the plane, Proc. Amer. Math. Soc. 9 (1958), 462-463.

Florida State University

${ }^{8}$ See also [4] and [5]. 